\title{
Los Mapas de Gestión de las Empresas Cotizadas Chilenas. Análisis Cuantitativo
}

\author{
Miguel A. Pérez-Benedito, Luis Porcuna-Enguix, Rubén Porcuna-Enguix \\ Facultad de Economía, Departamento de Contabilidad, Universidad de Valencia, Avenida dels Tarongers \\ s/n 46022 Valencia.-España (e-mail: miguel.a.perez@uv.es, luis.porcuna@uv.es, ruben.porcuna@uv.es)
}

Recibido Ene. 26, 2017; Aceptado Mar. 23, 2017; Versión final May. 22, 2017, Publicado Oct. 2017

\begin{abstract}
Resumen
Este artículo evalúa las clases de gestión adoptadas por las empresas localizadas en la región de Las Condes (Chile) a través de la metodología contable de los gráficos radiales. Esta metodología aplicada sobre dos empresas que utilizan dos diferentes clases de gestión, justifica las relaciones que existen entre los indicadores radiales y financieros. Los resultados contrastados con los precios de las acciones de las empresas analizadas en el mercado secundario confirman la validez de las relaciones anteriores. El análisis realizado ha permitido obtener generalizaciones sobre las distintas clases de gestión en la comuna de Las Condes, de acuerdo con el sentido multidimensional de los gráficos radiales, y justifica las estructuras financieras adoptadas por las empresas en el periodo de análisis. Se concluye que las clases de gestión existentes en una comuna actúan como un factor adicional en el estudio de la localización de las empresas.
\end{abstract}

Palabras clave: mapas de gestión; metodología contable; factor cultural

\section{Management Maps of Chilean Listed Companies. Quantitative Analysis}

\begin{abstract}
This manuscript assesses the kinds of management adopted by companies located in the municipality of Las Condes (Chile) through the use of the methodology of radar charts. This novel procedure, which is applied on two companies with two different kinds of management processes, justifies the relationship between radial and financial indicators. The results, contrasted with their stock prices on secondary market, confirm the validity of the relations mentioned above. The analysis allows generalizations regarding the different kinds of management in the municipality of Las Condes, according to the multidimensional sense of radar charts, and it justifies the adopted financial structures by companies in period of analysis. This study concludes that the existing kinds of management within a region act as an additional factor on companies' location research.
\end{abstract}




\section{INTRODUCCION}

El artículo se presenta como continuación de otro que tiene como antecedente el estudio cualitativo de las empresas cotizadas chilenas publicado en esta misma revista (Pérez et al., 2017). Este artículo tiene por objetivo analizar la situación patrimonial de las empresas a través de indicadores deducidos por aplicación de la teoría del seno y coseno sobre los gráficos radiales generados al incorporar los periodos medios de maduración de las empresas en cada uno de sus ejes. Se trata de analizar una imagen geométrica que presenta el resultado de la gestión realizada por las compañías en un periodo de tiempo. De esta forma, los indicadores obtenidos tienen valor positivo, en cualquier caso, se obtienen de forma independiente a la acción del medidor, están dotados de objetividad para medir la acción de la compañía ya que el analista no interviene en su composición y los resultados obtenidos pueden controlables en todo momento, al obtenerse de forma normalizada bajo la aplicación sistemática de una misma metodología.

Los antecedentes de esta metodología se encuentran en el artículo realizado por Martínez y Pérez (2011) y sus características no entran en contradicción con las adaptaciones normativas que establecen los mercados, al utilizar las variables que intervienen en las transacciones contables del ejercicio corriente para alcanzar un juicio de valor sobre la situación financiera de las entidades y, en contraposición a los análisis unidireccionales, la representación multidireccional de la actividad realizada permite observar el efecto de las decisiones adoptadas por las empresas en cada una de las áreas de gestión representadas simultáneamente en un gráfico radial. (Lantto y Sahlström, 2009; Terzi et al., 2013; Dimitrios et al., 2013; Ibiamke y Ateboh-Briggs, 2014; Buchman et al, 2016)

La obtención de gráficos radiales similares en un territorio geográfico determinado, independientemente del sector al que pertenecen las compañías localizadas en el mismo, indica que las situaciones patrimoniales de las empresas pueden ser analizadas considerando la clase contable de gestión que adoptan, dando lugar a los mapas contables de gestión. En consecuencia, la obtención de grupos homogéneos de gestión favorece orientar la realización de cualquier análisis de la actividad económica empresarial bajo un criterio causa-efecto frente a la utilización del criterio prueba-error en la selección de indicadores que justifiquen la acción económica de una población seleccionada para desarrollar una investigación determinada. El problema de investigación en el análisis de poblaciones o selección de muestras utilizando un criterio causa-efecto, justifica que este artículo presente la hipótesis de que existe una relación entre la clase contable de gestión adoptada por las empresas, en un momento del tiempo, y sus estructuras financieras.

La investigación desarrollada por Pérez $(2013,2014$ y 2015) justifica la hipótesis de que las empresas adoptan una determinada clase de gestión en función del entorno económico donde éstas desarrollan su actividad y todo ello sin considerar el sector económico al que pertenecen. Los efectos de los entornos económicos son considerados en las teorías de Williamson (Cárdenas y Ojeda, 2008; Salgado, 2003; Geyskens et al, 2006; Urbano et al., 2007; García y Taboada, 2012) y Krugman (Ascani et al., 2012; Sobrino, 2016) admitiendo la existencia de factores culturales tanto en las transacciones como en las decisiones de localización de las empresas. El efecto de la localización de las empresas en la cultura empresarial se ha puesto de manifiesto en el artículo de Pérez et al (2017), con el que se complementa éste ya citado anteriormente, al considerar estudios adicionales que analizan la existencia de conglomerados económicos identificados por adoptar las empresas similares estructuras organizativas, el hecho de que la calidad de las auditorias dependen del desarrollo económico e institucional de la provincia donde se encuentran localizadas las empresas auditadas e indican que la distancia entre las empresas afecta al flujo de bienes y servicios, a la toma de decisiones y al comportamiento del consumidor.

El artículo, además de esta parte introductora, presenta la metodología contable de los gráficos radiales en el segundo apartado. El siguiente apartado contiene un análisis de la gestión desarrollada por dos empresas localizadas en la región de las Condes, comparando los resultados obtenidos por aplicación de la metodología contable de los gráficos radiales con sus indicadores financieros y bursátiles. El cuarto apartado presenta el mapa de gestión chileno, indicando en cada región la clase de gestión adopta por las empresas localizadas en las mismas, y justifica la selección de la muestra realizada para alcanzar las conclusiones finales. El quinto apartado estudia el mapa de gestión de la región de Las Condes analizando las relaciones entre la situación financiera de las empresas localizadas en la misma y las clases de gestión que adoptan. Por último, se presentan las conclusiones alcanzadas en el estudio junto las limitaciones que se han presentado en la investigación y las líneas alternativas de investigación que permite desarrollar la metodología contable de los gráficos radiales.

\section{LA METODOLOGIA CONTABLE DE LOS GRAFICOS RADIALES}

La metodología de los gráficos radiales permite desarrollar tanto un análisis cualitativo como cuantitativo. El primer análisis analiza las clases de gestión como agrupaciones formales adoptadas por las empresas en 
un entorno económico. En análisis cualitativo del comportamiento de las empresas ha dado lugar a desarrollar una metodología propia de investigación basada en capacidad explicativa del indicador Chi2, contenida en un artículo de esta revista ya citado anteriormente. El análisis cuantitativo, que se desarrolla en este artículo, sirve para identificar las estructuras financieras que adoptan las entidades en un entorno económico y deducir la clase de financiación vinculada a la clase de gestión adoptada.

El desarrollo y justificación de la hipótesis planteada en el párrafo anterior se basa en la significación de los ángulos internos de los gráficos radiales que representan clases de gestión adoptadas por las empresas en la realización de su actividad. En la Fig. 1 están representadas tres áreas de gestión de las empresas Besalco SA (BSA) y Pacífico V Región SA (PVR). Estas empresas están localizadas en la región chilena de Las Condes y han mantenido sus clases de gestión durante el periodo de estudio comprendido entre 2005 y 2013. Las clases contables de gestión de ambas empresas se han generado a partir de incorporar sus respectivos periodos medios de maduración en los ejes de un gráfico radial, de acuerdo con la información obtenida de sus cuentas anuales en la base de datos Orbis, bajo licencia de la Universitat de Valencia. En la Fig. 1, se distinguen tres áreas de gestión asociadas a las distancias existentes entre sus respectivos periodos medios de maduración.

Las empresas BSA y PVR adoptan diferentes clases de gestión en la Fig. 1 y presentan mayor o menor concentración, en sus respectivos gráficos radiales, en los dos periodos anuales representados. Las clases contables de gestión se identifican por la diferente longitud de las distancias perimetrales DP1 y DP3 y la mayor o menor concentración de los gráficos radiales determina la menor o mayor dinámica de la actividad empresarial. En este sentido, la empresa PVR tiene una actividad más dinámica que la empresa BSA, en los dos años representados, y ambas compañías presentan mayor actividad dinámica en el año 2008 que en el año 2013, cuando las distancias perimetrales están menos dilatadas. Por otra parte, ambas compañías presentan las características de mantener estable la colocación de sus productos en el mercado y tener mayor dilatación en sus respectivas distancias perimetrales DP2 en el año 2013 que en el año 2008. El efecto observado manifiesta la diferencia de exigencia de liquidez en el área financiera de las empresas sobre las transacciones realizadas en el entorno económico donde están localizada las empresas.

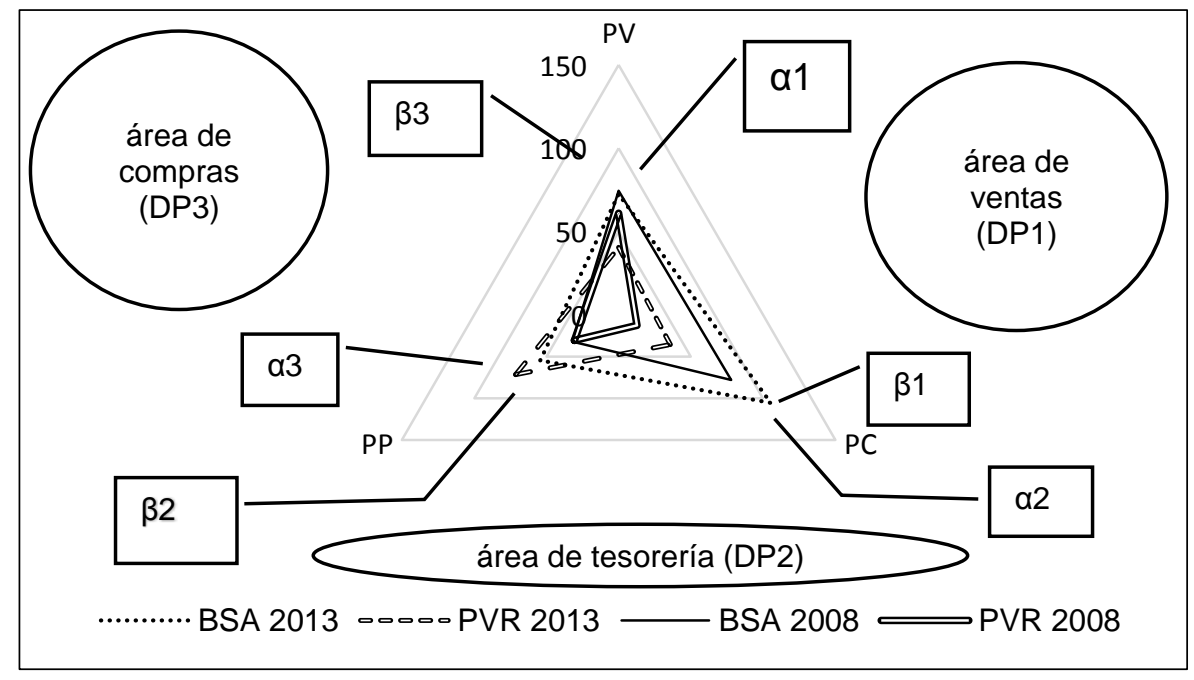

Fig. 1: Gráficos radiales de las empresas Pacífico V Región SA (PVR) y Besalco SA (BSA) en los años 2008 y 2013.

Con objeto de analizar el significado económico y financiero de las alteraciones de las distancias perimetrales, la metodología contable de los gráficos radiales analiza la alteración de los ángulos internos, ai y $\beta$ i de la Fig. 1, aplicando el teorema del coseno y del seno de acuerdo con las clases de gestión desarrollada por las empresas. El interés del estudio en el análisis de la variación de los ángulos internos reside en el hecho de que la alteración de un periodo medio de maduración provoca que todos los ángulos internos varíen al mismo tiempo. De esta forma, una decisión realizada en un área de gestión repercute en el conjunto de las áreas representadas en un gráfico radial simultáneamente.

Las clases de gestión son los distintos gráficos radiales generados a través de la incorporación de los periodos medios de maduración en cada uno de los ejes de un gráfico radial. Para diferenciar las clases de gestión es necesario medir las distancias perimetrales existentes entre los respectivos ejes radiales o periodos medios de maduración. La expresión (1) representa la aplicación del teorema del coseno y permite obtente las distancias perimetrales de cada una de las áreas representadas en la Fig.1. 


$$
D P_{k}^{2}=p_{i}^{2}+p_{j}^{2}-2 \times \cos \left(120 \times p_{i} \times p_{j}\right)
$$

Donde:

k=1, 2 y 3; 1=Área de ventas; 2=Área de tesorería; 3= Área de compras.

$\mathrm{i} \neq \mathrm{j} ; \mathrm{i}=\mathrm{j}=\mathrm{v}$ (ventas), c (cobro), $\mathrm{p}$ (pago)

La permuta sin repetición de las distancias perimetrales son las distintas clases de gestión que se pueden representar en un gráfico radial de tres dimensiones, se encuentran desarrolladas en la tabla $1 . \mathrm{El}$ calificativo de positivas o negativas que reciben las clases de gestión en la tabla 1, hace referencia a la calificación de las diferencias que se producen entre los periodos de pago y de cobro en el análisis contable. Sin embargo, este término conceptual se introduce para explicar la diferencia entre las distancias DP1 y DP3 de un gráfico radial. Cuando la distancia perimetral del área de compras (DP3) es superior (inferior) a la distancia perimetral del área de ventas (DP1), reciben el calificativo de positivas (negativas). Ambas distancias se miden desde el periodo de ventas. En consecuencia, las empresas que utilizan clases contables de gestión positivas obtienen mayor aplazamiento en la liquidación de sus operaciones con los proveedores que aquellas que utilizan clases de gestión negativas, cuando la distancia DP1 es mayor que la distancia perimetral DP3. Por otra parte, el valor de la distancia perimetral DP2 permite discriminar entre las clases de gestión que presentan mayor o menor tensión en la liquidación de las operaciones comerciales. El valor de la distancia DP2 está asociado a la mayor o menor liquidez del mercado en el que actúan las empresas localizadas en un mismo entorno económico. Este aspecto justifica el comportamiento común de las compañías BSA y PVR en los años 2008 y 2013 en la Fig. 1. El análisis comparado entre los años 2008 y 2013, justifica que en el año 2013, cuando la crisis financiera se presenta más acentuada, las distancias perimetrales DP2 se encuentran más dilatadas. El análisis de los efectos de la crisis financiera y los cambios de gestión pueden observase en el artículo de Pérez y otros (2017) presentado en esta misma revista, a través del análisis cualitativo realizado sobre la misma muestra de empresas. Las distintas clases de gestión se presentan en la tabla 1, de acuerdo con la comparación de las distancias perimetrales DP3 y DP1, como se ha indicado anteriormente.

Tabla 1: Clases de Gestión en un gráfico radial de tres dimensiones.

\begin{tabular}{|l|c|l|c|}
\hline $\begin{array}{l}\text { HOLGURAS FINANCIERAS POSITIVAS } \\
\text { (DP3>DP1) }\end{array}$ & \multicolumn{2}{|c|}{$\begin{array}{c}\text { HOLGURAS FINANCIERA NEGATIVAS } \\
\text { (DP1>DP3) }\end{array}$} \\
\hline CLASE A & DP2>DP3>DP1 & CLASE D & DP1>DP3>DP2 \\
\hline CLASE B & DP3>DP2>DP1 & CLASE E & DP1>DP2>DP3 \\
\hline CLASE C & DP3>DP1>DP2 & CLASE F & DP2>DP1>DP3 \\
\hline
\end{tabular}

Las combinaciones de las distancias perimetrales indican las clases de gestión adoptadas por las empresas y el resultado de su gestión puede analizarse a través de la significación económica y financiera de los ángulos internos de los gráficos radiales. Para alcanzar este último objetivo, se aplica el teorema del seno, de acuerdo con la expresión 2 , en cada clase de gestión.

$\frac{\mathrm{DP}_{\mathrm{k}}}{\operatorname{seno}(120)}=\frac{\mathrm{p}_{\mathrm{i}}}{\operatorname{seno}\left(\alpha_{\mathrm{k}}\right)}=\frac{\mathrm{p}_{\mathrm{j}}}{\operatorname{seno}\left(\beta_{\mathrm{k}}\right)}$

Donde:

k=1, 2 y 3. 1=Área de ventas; 2=Área de tesorería; 3=Área de compras.

$\mathrm{i} \neq \mathrm{j} ; \mathrm{i}=\mathrm{j}=\mathrm{v}$ (ventas), c (cobro), $\mathrm{p}$ (pago)

La Tabla 2 contiene los resultados comparativos de las distancias perimetrales en las clases de gestión B y E, identificadas en la tabla 1. Estas clases de gestión son las adoptadas por las empresas PVR y BSA en el último año del análisis desarrollado en el artículo, en las que la distancia DP2 adopta una posición intermedia. Para realizar la evaluación de las clases de gestión se diferencian entre condiciones necesarias y condiciones suficientes. Las primeras deben cumplirse necesariamente para identificar cada clase de gestión. Las condiciones suficientes miden tanto la suficiencia financiera obtenida por las empresas en el ejercicio de su actividad como la liquidez en las transacciones realizadas. El contraste entre las condiciones de suficiencia financiera y liquidez de transacción indica en qué medida la actividad empresarial desarrollada por las compañías favorecen su posición financiera. Una posición financiera favorable para la compañía se obtiene cuando la condición de suficiencia financiera y liquidez de transacción se cumplen al mismo tiempo. Cuando ambas condiciones suficientes se cumplen, la financiación obtenida por la empresa 
en el ejercicio de su actividad empresarial es consistente con la interacción entre las partidas del resultado contable y del balance que se encuentran vinculadas en cada una de las transacciones contables, en un área de gestión. Es decir, la empresa obtiene liquidez en la realización de las transacciones en un área concreta. En caso contrario, las transacciones realizadas se han financiado de forma ajena a la actividad empresarial. De acuerdo con lo indicado, las situaciones patrimoniales de las empresas son consecuencia del resultado de las transacciones contables realizadas, cuya liquidez se mide a través de la metodología contable de los gráficos radiales. En consecuencia, la metodología contable de los gráficos radiales permite medir la acción empresarial en cada una de las áreas de gestión representada en un gráfico radial, calificar la clase de gestión realizada en su conjunto y conocer su efecto en la estructura financiera de la empresa.

Tabla 2: Condiciones para evaluar la gestión de las holguras financieras positivas

\begin{tabular}{|l|c|c|}
\hline CLASE DE GESTION (CG) & CG_B & CG_E \\
\hline CONDICIONES NECESARIAS & DP3>DP2>DP1 & DP1>DP2>DP3 \\
\hline$\alpha 1$ & $\alpha 1<30$ & $\alpha 1>30$ \\
\hline$\beta 1$ & $\beta 1>30$ & $\beta 1<30$ \\
\hline$\alpha 2$ & $\alpha 2>30$ & $\alpha 2<30$ \\
\hline$\beta 2$ & $\beta 2<30$ & $\beta 2>30$ \\
\hline$\alpha 5$ & $\alpha 3<30$ & $\alpha 3>30$ \\
\hline$\beta 3$ & $\beta 3>30$ & $\beta 3<30$ \\
\hline CONDICIONES SUFICIENTES & & $\beta 3 / \alpha 2>1$ \\
\hline SUFICIENCIA FINANCIERA VENTAS & $\alpha 2 / \beta 3>1$ & $1>C V /{ }^{*} \mathrm{CL} / \mathrm{EX}>\beta 2 / \alpha 3$ \\
\hline TRANSACCIÓN EN VENTAS & $1>\beta 2 / \alpha 3>\mathrm{CV} / \mathrm{N}^{*} \mathrm{CL} / \mathrm{EX}$ & $\alpha 3 / \beta 1>1$ \\
\hline SUFICIENCIA FINANCIERA TESORERIA & $\beta 1 / \alpha 3>1$ & $1>\mathrm{C} / \mathrm{N}^{*} \mathrm{CL} / \mathrm{PR}>\alpha 1 / \beta 3$ \\
\hline TRANSACCIÓN EN TESORERIA & $1>\alpha 1 / \beta 3>\mathrm{C} / \mathrm{V}^{*} \mathrm{CL} / \mathrm{PR}$ & $\beta 2 / \alpha 1>1$ \\
\hline SUFICIENCIA FINANCIERA COMPRAS & $\alpha 1 / \beta 2>1$ & $1>\mathrm{C} / \mathrm{CV}{ }^{*} \mathrm{EX} / \mathrm{PR}>\beta 1 / \alpha 2$ \\
\hline TRANSACCIÓN EN COMPRAS & $1>\beta 1 / \alpha 2>\mathrm{C} / \mathrm{CV}{ }^{*} \mathrm{EX} / \mathrm{PR}$ & \\
\hline
\end{tabular}

Las condiciones necesarias y suficientes establecidas en la Tabla 2 son obtenidas por aplicación de las expresiones (1) y (2) sobre las clases de gestión B y E. Las condiciones necesarias son medidas a través de valores absolutos de los ángulos internos de los triángulos de cada área de gestión y las condiciones suficientes son medidas a través de sus relaciones. Estas últimas se diferencian entre financieras y liquidez de transacciones. Las condiciones de suficiencia financiera miden la capacidad de financiación que obtiene la empresa de acuerdo con los periodos medios de maduración de las transacciones contables realizadas. La suficiencia financiera del área de ventas es medida a través de relación de ángulos con diferente índice al del área que evalúan y, de la misma forma, ocurre con el resto de condiciones de esta misma naturaleza en las otras clases de gestión. Las condiciones de liquidez de transacción comparan la interacción entre las variables contables que intervienen en las transacciones y las relaciones angulares generadas por la actividad empresarial en las distintas áreas de gestión. De esta forma, en el área de ventas, la interacción entre las cuentas de Coste de ventas (CV) y Ventas (V), que intervienen en la obtención del resultado empresarial, y los valores medios de las cuentas de Clientes (CL) y Existencias (Ex), pertenecientes al balance de las entidades, es comprada con la relación angular de los ángulos opuestos a la distancia perimetral (DP1) del área que se pretende evaluar, como puede observarse en la Fig. 1. La participación de cuentas en las interacciones de las otras dos áreas son la media simple de la cuenta de Proveedores (PR) y el valor de la cuenta de Compras (C). El contraste entre las condiciones de suficiencia financiera y liquidez de transacción permite observar en qué medida el ejercicio de la actividad empresarial condiciona la estructura financiera de las empresas.

\section{APLICACIONES METODOLÓGICAS.}

En la metodología contable de los gráficos radiales, la gestión realizada por una empresa es óptima cuando las áreas de gestión representadas en un gráfico radial cumplen las condiciones de suficiencia financiera y liquidez de transacción. Cuando se cumplen las condiciones de suficiencia financiera y no lo hacen las de liquidez de la transacción, las fuentes de financiación ajenas a la actividad empresarial permiten desarrollar la gestión continuada de la actividad, de acuerdo con las condiciones de transacción establecidas en el mercado. Si dejan de cumplirse ambas condiciones, la empresa debe cambiar su gestión en el área afectada, y cuando esta característica se cumple en las tres áreas de gestión al mismo tiempo, la empresa puede cesar en su actividad. Como ejemplo ilustrativo de lo indicado anteriormente, se analiza el resultado de la actividad empresarial realzada por las empresas de la Fig. 1. Las tablas 3 y 4 contienen, respectivamente, los resultados obtenidos para la empresa BSA y PVR por aplicación de la metodología contable de los gráficos radiales. 
La estructura de las tablas 3 y 4 presenta el resultado de las relaciones angulares que miden las condiciones de suficiencia financiera (SF) y liquidez de transacción (LT) en cada una de las áreas de gestión representadas en los gráficos radiales de las empresas BSA y PVR. Las tablas se completan con el resultado de la comparación entre las condiciones indicadas a través del indicador de condición de liquidez (CL), para las correspondientes áreas de gestión, y con los indicadores financieros que miden el efecto de las decisiones adoptadas en la estructura financiera de las empresas. El indicador CL toma el valor 1 si se cumplen las condiciones de SF y LT, en caso contrario toma el valor cero. Su valor tiene efectos en la estructura financiera de las empresas que es medida a través del ratio de autonomía financiera (RAF), el cual compara el volumen de fondos propios de la empresa con el total de las fuentes de financiación externa, y los que determinan la importancia relativa de cada una de las fuentes de financiación respecto del total del pasivo del balance.

En la tabla 3 se presentan los resultados obtenidos en la evaluación de la actividad de la empresa BSA. La empresa concede mayor aplazamiento de cobro a los clientes que el obtenido de los proveedores, provocando que la distancia DP1 sea superior a la distancia DP3. Esta clase de gestión indica que se exige menor liquidez de las transacciones contables en el área de ventas que en el área de compras, medida desde la colocación del producto en el mercado. Los resultados obtenidos muestran que la empresa no obtiene liquidez en sus respectivas áreas de gestión y, sin embargo, cumplen las condiciones de suficiencia financiera. En consecuencia, la financiación ajena a la actividad empresarial es la que mantiene la actividad empresarial y es la compañía la que concede liquidez al mercado aumentando sus fondos propios. Este efecto se produce en la compañía BSA durante el periodo comprendido entre 2006 y 2012. Así, en el año 2009 la empresa aumenta su Capital en un 150\% y esta decisión, sin ir acompañada de un cambio de gestión, no mejora la situación financiera de la compañía en los años sucesivos. La participación de los fondos propios en la estructura financiera de la empresa se mantiene superior a las otras fuentes de financiación en el periodo indicado. Las dificultades de liquidez se reproducen en los años 2013 y 2014 , donde el exigible a corto plazo aumenta su presencia en la estructura financiera de la empresa de forma significativa, comparándolo con su participación en los años anteriores. En el año 2014 el exigible a corto plazo alcanza la misma participación que el exigible a largo plazo (29\%), e indica las dificultades de la empresa en el ejercicio de su actividad empresarial.

Tabla 3: Condiciones radiales y ratios financieros de la empresa BSA

\begin{tabular}{|l|c|c|c|c|c|c|c|c|c|}
\hline Años & 2014 & 2013 & 2012 & 2011 & 2010 & 2009 & 2008 & 2007 & 2006 \\
\hline Clase de gestión & $\mathrm{E}$ & $\mathrm{E}$ & $\mathrm{E}$ & $\mathrm{E}$ & $\mathrm{E}$ & $\mathrm{E}$ & $\mathrm{E}$ & $\mathrm{E}$ & $\mathrm{E}$ \\
\hline SF Ventas & 1,2159 & 1,2948 & 1,1333 & 1,0137 & 1,0919 & 1,1759 & 1,0351 & 1,0669 & 1,4654 \\
\hline LT Ventas & 1,0919 & 1,1662 & 1,0839 & 1,0096 & 1,0614 & 1,1030 & 1,0134 & 1,0212 & 1,1153 \\
\hline CL Ventas & 0 & 0 & 0 & 0 & 0 & 0 & 0 & 0 & 0 \\
\hline SF Tesorería & 1,5369 & 1,4528 & 1,3012 & 1,1859 & 1,2490 & 1,3614 & 1,4677 & 1,5637 & 1,9496 \\
\hline LT Tesorería & 1,6748 & 1,4270 & 1,3244 & 1,2219 & 1,2735 & 1,3856 & 1,8032 & 2,0672 & 2,3414 \\
\hline CL Tesorería & 0 & 0 & 0 & 0 & 0 & 0 & 0 & 0 & 0 \\
\hline SF Compras & 1,2593 & 1,1196 & 1,1471 & 1,1697 & 1,1433 & 1,1561 & 1,4166 & 1,4624 & 1,3119 \\
\hline LT Compras & 1,5279 & 1,2210 & 1,2208 & 1,2102 & 1,1993 & 1,2544 & 1,7776 & 2,0198 & 2,0701 \\
\hline CL Compras & 0 & 0 & 0 & 0 & 0 & 0 & 0 & 0 & 0 \\
\hline RAF & 0,6785 & 0,8586 & 1,2559 & 1,1392 & 0,8469 & 1,4507 & 0,8379 & 0,9769 & $-0,1376$ \\
\hline Fondos propios (\%) & $40,42 \%$ & $46,20 \%$ & $55,67 \%$ & $53,25 \%$ & $45,85 \%$ & $59,20 \%$ & $45,59 \%$ & $49,41 \%$ & $-15,95 \%$ \\
\hline Exigible I.pl. (\%) & $29,87 \%$ & $30,61 \%$ & $29,24 \%$ & $29,27 \%$ & $40,81 \%$ & $31,58 \%$ & $43,49 \%$ & $41,76 \%$ & $101,70 \%$ \\
\hline Exigible c.pl. (\%) & $29,71 \%$ & $23,19 \%$ & $15,09 \%$ & $17,48 \%$ & $13,34 \%$ & $9,22 \%$ & $10,92 \%$ & $8,82 \%$ & $14,25 \%$ \\
\hline TOTAL & $100 \%$ & $100 \%$ & $100 \%$ & $100 \%$ & $100 \%$ & $100 \%$ & $100 \%$ & $100 \%$ & $100 \%$ \\
\hline
\end{tabular}

Los resultados obtenidos a través del análisis radial en la Tabla 3, pueden ser contrastados con la evolución de las acciones de la sociedad BAS (Besalco SA) en el mercado de valores de Santiago de Chile, representada a través de gráficos de vela en la Fig. 2. En el eje secundario de las ordenadas de la Fig.2 se encuentran representados los valores medios de las acciones Besalco SA en la Bolsa de Chile consultada en España el16/5/2016 a las 5:24, correspondientes al precio de apertura, precio máximo, precio mínimo y precio de cierre, y en el eje primario se encuentran representados los volúmenes de contratación. La evolución de las variables del mercado de valores representadas en la Fig. 2 se presenta acorde con las dos crisis financieras. En la primera crisis, los niveles bajos de contratación y baja cotización están de acuerdo con el nivel de riesgo existen en el mercado del año 2008. En el periodo de 2006 a 2010, la perdida de suficiencia financiera y el mejor comportamiento del indicador de liquidez de transacción en las tres áreas de gestión, hace que las cotizaciones de la sociedad sean elevadas en 2010. El año 2011 supone un cambio en los resultados de gestión. La tendencia continuada desde 2006 se interrumpe con un aumento de la suficiencia financiera en el área de compras y una pérdida de liquidez en las condiciones de transacción en esa área. Esta tendencia continúa en los años sucesivos en todas las áreas y afecta a la pérdida de 
cotización de las acciones. Es decir, como se ha anticipado, la condición de liquidez de transacción determina la calidad de la suficiencia financiera obtenida. En los años 2012 a 2014, últimos años en la tabla 3 , la suficiencia financiera se incrementa en la misma medida que lo hacen las condiciones de liquidez en las tres áreas. En consecuencia, el aumento de la suficiencia financiera de la empresa Besalco SA no está justificado con los resultados de liquidez de las transacciones realizadas en los años 2012 a 2014. Es posible observar como su cotización disminuye en la Fig.2 y el peso de la financiación propia no alcanza el $50 \%$ de las fuentes de financiación en 2014 , poniendo en riesgo la solvencia de la entidad.

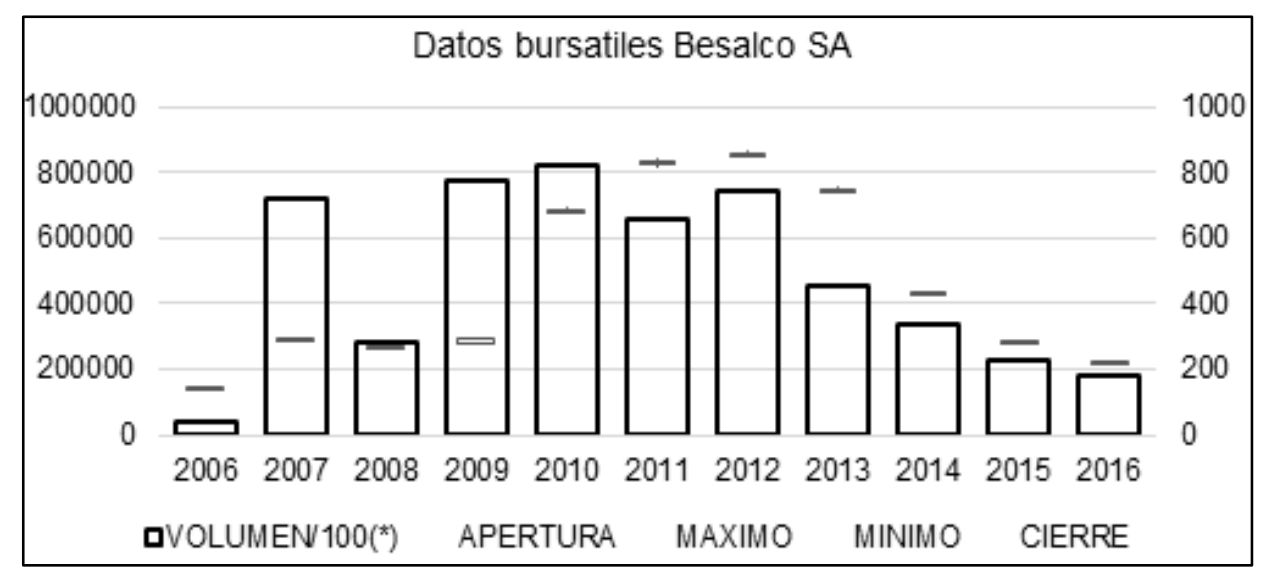

Fig. 2: Acciones Besalco. Fuente: Bolsa de Santiago.

La clase de gestión adoptada por la compañía Besalco S.A., en el entorno económico donde desarrolla su actividad, no permite obtener óptimos de gestión. El incremento de las suficiencias financieras en los últimos años y el deterioro de las condiciones de liquidez de transacciones, supone corregir el efecto de interacción entre las partidas de resultados y de balance. Los informes financieros de Besalco SA en junio de 2014 y marzo de 2016, facilitados por la Bolsa de Santiago, muestran las variaciones de las Ganancias Brutas y de los Pasivos Corrientes. En junio de 2014 presenta un incremento de Ganancia Bruta de un 12\% y un incremento de los Pasivos corrientes de $61 \%$, respecto del periodo anterior. En el informe de marzo de 2016, los incrementos de las Ganancias Brutas y del Pasivo corriente, fueron respectivamente de un $4 \%$ y de un 1\%, respecto del año anterior. La obtención de un incremento del 30\% en las Ganancias del Periodo de 2016 frente a la disminución de un 92\% en 2014, suponen una reestructuración de la actividad relevante. Sin embargo, las deudas acumuladas hacen que la importancia relativa del Neto patrimonial sea del $27,59 \%$ del total del pasivo en marzo de 2016 y presenten la segunda estimación valorativa media de las acciones de la sociedad Besalco SA más baja del periodo analizado. Realizada una consulta en la Bolsa de Chile el 16 de mayo de 2016 , el valor de la acción era de $248 \$$ frente al precio mínimo de $202 \$$, premiando el mercado las decisiones de gestión adoptadas.

Tabla 4: Condiciones radiales y ratios financieros de la empresa PVR

\begin{tabular}{|l|c|c|c|c|c|c|c|c|c|}
\hline YEAR & 2013 & 2012 & 2011 & 2010 & 2009 & 2008 & 2007 & 2006 & 2005 \\
\hline AMG & $\mathrm{B}$ & $\mathrm{B}$ & $\mathrm{B}$ & $\mathrm{A}$ & $\mathrm{B}$ & $\mathrm{C}$ & $\mathrm{C}$ & $\mathrm{C}$ & $\mathrm{C}$ \\
\hline SF Ventas & 1,0446 & 1,1495 & 1,1141 & 1,0232 & 1,4461 & 2,2635 & 2,7907 & 2,4855 & 1,6315 \\
\hline LT Ventas & 0,9165 & 0,7184 & 0,8362 & 1,0319 & 0,4775 & 0,4067 & 0,5888 & 0,4314 & 0,7025 \\
\hline CLT Ventas & 0 & 0 & 1 & 0 & 0 & 1 & 1 & 1 & 1 \\
\hline SF Tesorería & 1,5303 & 1,7790 & 1,3925 & 1,1707 & 1,6342 & 1,2417 & 1,0148 & 1,1743 & 1,0280 \\
\hline LT Tesorería & 0,7166 & 0,5864 & 0,7267 & 0,8787 & 0,4586 & 0,4853 & 0,9354 & 0,5446 & 0,9406 \\
\hline CLTesorería & 1 & 1 & 1 & 1 & 0 & 1 & 0 & 1 & 0 \\
\hline SF Compras & 1,4629 & 1,5370 & 1,2479 & 1,1979 & 1,1253 & 1,7848 & 2,7409 & 2,0698 & 1,5851 \\
\hline LT Compras & 0,7829 & 0,8218 & 0,8704 & 0,8514 & 0,9647 & 1,1683 & 1,5835 & 1,2344 & 1,3373 \\
\hline CLC & 1 & 1 & 1 & 1 & 1 & 0 & 0 & 0 & 0 \\
\hline RAF & 1,985 & 1,516 & 0,867 & 1,790 & 1,799 & 1,006 & 1,017 & 1,041 & 0,940 \\
\hline Fondos propios (\%) & $66,50 \%$ & $60,25 \%$ & $46,44 \%$ & $64,16 \%$ & $64,28 \%$ & $50,16 \%$ & $50,41 \%$ & $51,00 \%$ & $48,46 \%$ \\
\hline Exigible I.pl. (\%) & $12,68 \%$ & $15,40 \%$ & $17,28 \%$ & $18,47 \%$ & $19,07 \%$ & $14,79 \%$ & $14,72 \%$ & $15,89 \%$ & $25,95 \%$ \\
\hline Exigible c.pl. (\%) & $20,82 \%$ & $24,35 \%$ & $36,28 \%$ & $17,37 \%$ & $16,65 \%$ & $35,05 \%$ & $34,86 \%$ & $33,11 \%$ & $25,58 \%$ \\
\hline \multicolumn{1}{c}{ TOTAL } & $100 \%$ & $100 \%$ & $100 \%$ & $100 \%$ & $100 \%$ & $100 \%$ & $100 \%$ & $100 \%$ & $100 \%$ \\
\hline
\end{tabular}

En la Tabla 4 se presentan los resultados obtenidos en la evaluación de la actividad de la empresa PVR. La empresa PVR dirige su gestión hacia el área de compras, obteniendo un mayor aplazamiento en las 
liquidaciones de las operaciones de compras que de ventas. La empresa PVR (PACIFICO V REGION SA) presenta un cambio de gestión en el año 2009, adoptando la clase B de gestión. Desde el principio del periodo, la liquidez de la actividad empresarial se obtiene en el área de ventas y esta situación cambia en 2009. La liquidez de las transacciones se obtiene en el área de compras y en el área de tesorería al mismo tiempo. La clase de gestión B se mantiene hasta el final del periodo y se alcanza un óptimo en el año 2011, cuando las tres áreas alcanzan suficiencia financiera y se cumple la liquidez de transacción en las tres áreas. La estructura financiera en 2011 es similar a la obtenida en los años 2006, 2007 y 2008. El cambio de gestión en 2009 presenta una estructura financiera similar a la de los años posteriores a 2009, excepto el año 2011. La estructura financiera de la empresa ofrece garantías al mercado financiero para el ejercicio continuado de la actividad. Esta garantía significa alcanzar un mayor peso de los fondos propios en la estructura financiera de la empresa, que se incrementa cuando el área de compras empieza a cumplir la condición de liquidez del resultado junto con la del área de tesorería. Es decir, la liquidez que obtiene la empresa le permite reducir su pasivo exigible y neto patrimonial alcanza mayor importancia en la estructura financiera de la empresa.

El análisis comparado de la evolución de las dos sociedades, puesto de manifiesto a través de la evolución de la importancia relativa del exigible a corto plazo (Exigible c.pl.), tiene significación diferente en ambas compañías. La empresa BSA dirige su gestión hacia el área de ventas, concediendo crédito al mercado, dado que la distancia perimetral DP1 es superior a la distancia DP3. El incumplimiento de la condición de liquidez de transacción en esta empresa, provoca utilizar fuentes financiación ajenas a la actividad y el riesgo de solvencia financiera de la empresa es elevado cuando la importancia relativa de los fondos propios es inferior a un $50 \%$. En contraposición, la empresa PVR obtiene crédito comercial del mercado y debe ofrecer garantías a los proveedores (mercado) cuando su distancia perimetral DP3 del área de compras es suprior a la distancia perimetral DP1 del área de venas. Es decir, existe una menor tensión en la liquidación de las operaciones de compras que en las operaciones de ventas. Esta empresa cumple las condiciones liquidez de tesorería y de suficiencia financiera en los años 2008 y 2011, cuando el indicador Exigible a c.pl toma los valores más elevados de la serie temporal. En los periodos de crisis financiera, la suficiencia financiera aumenta respecto de los años anteriormente citados y permite mantener la garantía de solvencia en el mercado cuando el peso relativo de los fondos propios en la tabla 4 (Fondos propios (\%)) es superior al 50\% en 2008 y alcanza, en los años posteriores a 2011, niveles superiores al $60 \%$. El cambio de gestión de la clase $\mathrm{C}$ a la clase $\mathrm{B}$ en esta empresa, justifica los cambios producidos en la estructura financiera. La consulta realizada en la Bolsa de Chile para la empresa Pacifico V Región SA, en la misma fecha que para realizada para la empresa Besalco SA, presenta un precio de la acción de 590\$, cuando su menor valor alcanzado en el año 2016 es de 589,99\$. La opinión comparada del mercado respecto de las clases de acciones confirma el análisis singular realizado sobre las dos sociedades localizadas en la región de Las Condes.

En este apartado, hemos observado como las alteraciones en la estructura financiera de las empresas se obtienen o son resultado de las decisiones de gestión adoptadas. La medida de las decisiones de gestión tiene efectos multidireccionales y su análisis diversificado, en cada una de las áreas de gestión representadas en un gráfico radial, dota de mayor capacidad explicativa de la respuesta de las empresas ante los sucesos del mercado en el que actúan. En consecuencia, los efectos compensados en la acción empresarial alcanzan mayor significación. La valoración comparada de las empresas BSA y PVR utilizando indicadores de la Bolsa de Chile, se ha realizado con objeto de considerar un criterio externo de opinión sobre los resultados de gestión realizados por un organismo institucional independiente que, aunque afectado por un riesgo sistémico, tiene una opinión homogénea sobre la evolución de la actividad desarrollada por las empresas cotizadas chilenas.

\section{LOS MAPAS DE GESTIÓN}

Los mapas de gestión son las distintas clases de gestión adoptadas por las empresas en una región, independientemente del sector al que pertenecen en un momento del tiempo. La formación de los mapas de gestión se ha realizado considerando la división administrativa del territorio de Chile. Las empresas elegidas se han obtenido utilizando la base de datos Orbis con licencia de la Universidad de Valencia en España y el criterio utilizado ha sido elegir como elementos de la muestra las empresas cotizadas en la bolsa de Chile, obteniendo como resultado 163 empresas que, excluyendo las entidades del sector financiero así como aquellas empresas de las que no se ha podido obtener información suficiente para la obtención de los periodos medios de maduración, han resultado 127 empresas. La selección de empresas cotizadas en la composición de la muestra se ha realizado bajo la hipótesis de que se aplican criterios homogéneos de contabilización de las transacciones. La primera discriminación realizada sobre la muestra de empresas obtenida ha permitido identificar dos regiones chilenas con significación en la localización de las empresas, Santiago con 79 empresas y Las Condes con 19. La Tabla 5 recoge las clases de gestión desarrolladas por las empresas chilenas durante el periodo de análisis atendiendo a la localización administrativas de las mismas. 
Tabla 5: Tabla de contingencia anual

\begin{tabular}{|c|c|c|c|c|c|c|c|c|c|c|c|c|c|c|c|}
\hline Total del periodo & A & B & C & D & E & $\mathrm{F}$ & SUMA & & A & B & C & D & E & $\mathrm{F}$ & SUMA \\
\hline${ }^{*}$ ANTOFAGASTA* & 1 & 0 & 0 & 0 & 0 & 8 & 9 & $\ldots / \ldots$ & $\ldots$ & $\ldots$ & $\ldots$ & $\ldots$ & $\ldots$ & $\ldots$ & $\ldots$ \\
\hline${ }^{*} \mathrm{ARICA}{ }^{*}$ & 2 & 0 & 0 & 0 & 0 & 7 & 9 & *LO BARNECHEA* & 6 & 0 & 0 & 0 & 6 & 6 & 18 \\
\hline${ }^{*} \mathrm{CONCEPCION}{ }^{*}$ & 4 & 0 & 0 & 0 & 0 & 0 & 4 & ${ }^{*} \mathrm{MACUL}^{*}$ & 2 & 0 & 0 & 0 & 0 & 5 & 7 \\
\hline${ }^{*} \mathrm{CONCON}^{*}$ & 1 & 0 & 0 & 0 & 0 & 4 & 5 & ${ }^{*} N U N O A *$ & 0 & 0 & 0 & 9 & 0 & 0 & 9 \\
\hline *HUECHURABA* & 0 & 0 & 0 & 0 & 1 & 5 & 6 & *OSORNO* & 0 & 0 & 0 & 0 & 0 & 9 & 9 \\
\hline *IQUIQUE* & 6 & 0 & 0 & 0 & 0 & 9 & 15 & ${ }^{*}$ PROVIDENCIA* & 19 & 0 & 0 & 0 & 1 & 5 & 25 \\
\hline${ }^{*}$ LA FLORIDA* & 0 & 0 & 0 & 10 & 0 & 0 & 10 & *PUERTOMONTT* & 2 & 2 & 2 & 1 & 0 & 0 & 7 \\
\hline *LA REINA* & 2 & 0 & 0 & 0 & 0 & 1 & 3 & *SANTIAGO* & 238 & 59 & 72 & 101 & 41 & 85 & 596 \\
\hline${ }^{\star}$ LA SERENA* & 0 & 0 & 0 & 0 & 1 & 8 & 9 & ${ }^{*}$ TALCA* & 3 & 0 & 0 & 0 & 0 & 6 & 9 \\
\hline *LAS CONDES* & 31 & 28 & 14 & 41 & 15 & 27 & 156 & ${ }^{*}$ VALDIVIA* & 4 & 0 & 0 & 0 & 2 & 3 & 9 \\
\hline *LO BARNECHEA* & 6 & 0 & 0 & 0 & 6 & 6 & 18 & *VALPARAISO* & 26 & 10 & 2 & 0 & 0 & 4 & 42 \\
\hline$\ldots / \ldots$ & $\ldots$ & $\ldots$ & $\ldots$ & $\ldots$ & $\ldots$ &.. & $\ldots$ & TOTAL & 347 & 99 & 90 & 162 & 67 & 192 & 957 \\
\hline
\end{tabular}

El resultado obtenido sobre las 20 regiones de la Tabla 5 muestra que, diferenciando entre clases contables de gestión positivas y negativas, existen 6 provincias que mantienen una misma clase de gestión durante el horizonte temporal de análisis, 12 de ellas adoptan preferentemente una de ellas, 8 negativas y 4 positivas, con una media superior al $70 \%$ del total de cada región y las regiones de Las Condes y Santiago concentran la mayoría de las clases contables de gestión, presentando un reparto equitativo entre positivas y negativas. La alternancia de las clases de gestión en la región de Las Condes, presentando un mayor número de clases de gestión negativas que positivas, frente al resultado general obtenido, ha sido el elemento significativo para analizar la actividad desarrollada en esa región.

\section{LAS CLASES DE GESTIÓN EN LA REGIÓN DE LAS CONDES.}

Las clases de gestión en la región chilena de la Las Condes se presentan en la Fig. 3, donde las burbujas sombreadas representan las empresas que han cambiado de clase de gestión negativa hacia positiva a lo largo del periodo ( $\left.\mathrm{CG}_{+-}\right)$. Etas últimas, las que mantienen una gestión positiva, están representadas con líneas continuas $(\mathrm{CG}++)$ y las empresas con clase de gestión negativa ( $\mathrm{CG}$ - -) están representadas con burbujas de líneas paralelas.

La distribución geográfica de las empresas localizadas en Las Condes ha sido elaborada considerando su latitud, longitud y altura utilizando un gráfico de burbujas, donde la mayor o menor amplitud de un círculo representa la mayor o menor altura respecto del nivel del mar. La información de estas variables se ha realizado utilizando la web http://www.coordenadas-gps.com/. La obtención del mapa de gestión de Las Condes se encuentra en la Fig. 3, donde la empresa BESALCO SA (BSA) tiente las siguientes coordenadas: latitud -33,416361, longitud -70,603001, altura 640 y la empresa PACIFICO V REGION SA (PVR) se encuentra localizada en latitud -33,418918, longitud -70,599273, altura 640. Su localización, próxima a la región de Santiago de Chile, indica que el efecto frontera no altera la clase de gestión adoptada por la compañía BSA, incidiendo en el interés del análisis de su localización.

La localización de las empresas la región de Las Condes tiene como principal característica mantener y cambiar las clases de gestión en función de las perturbaciones en el mercado. Las clases de gestión adoptadas no dependen del sector al que se encuentre vinculada la industria, sino más bien de las condiciones del entorno económico donde la empresa desarrolla su actividad. En general, las empresas localizadas próximas a los centros de decisión gubernamentales adoptan clases de gestión positivas, las empresas alejadas de los centros de decisión adoptan clases de gestión negativas y los espacios geográficos donde las empresas adoptan clases de gestión positivas y negativas se identifica con áreas geográficas con nivel socioeconómico alto y medio alto y no incide sobre la clase de gestión adoptada. La región de las Condes es conocida como Sanhattan o la Manhattan chilena, presenta la característica de ser el área comercial más activa de Chile y las empresas tienen flexibilidad en adoptar las clases de gestión diversificadas. 


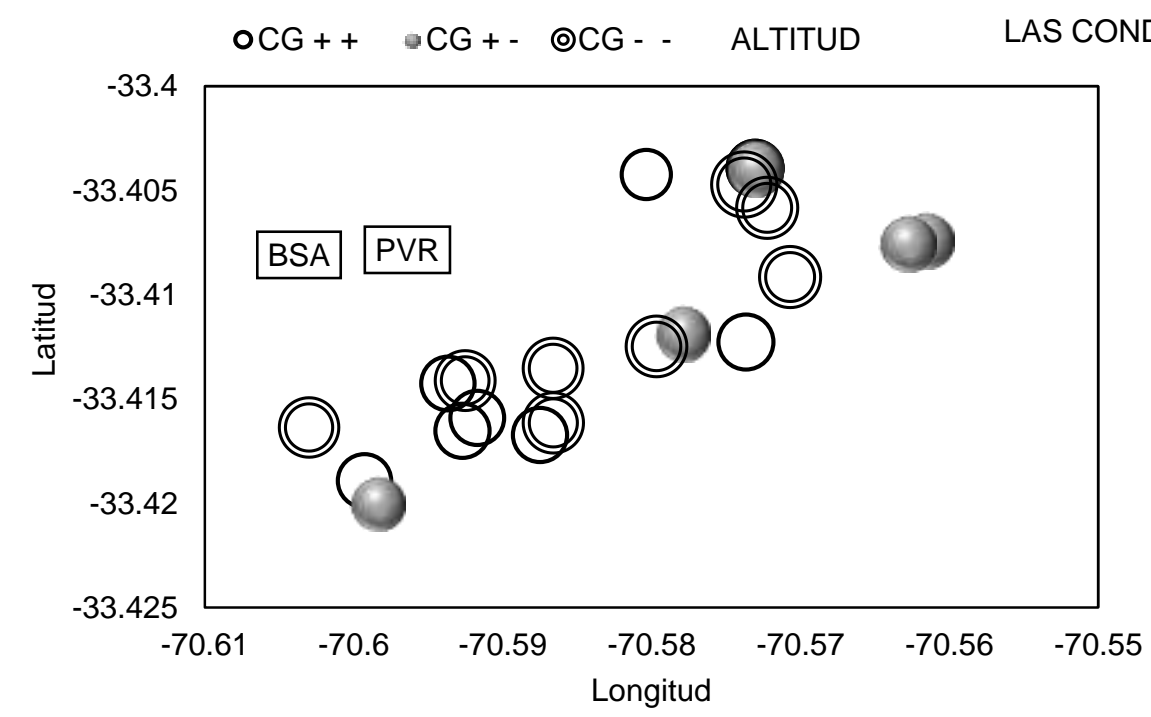

Fig. 3: Mapa geográfico de gestión de la región Las Condes.

Las consecuencias de adoptar una clase de gestión u otra ha sido medida a través de indicadores angulares que evalúan la clase de gestión adoptada por una empresa en sentido multidireccional, como el caso de BSA y PVR en el apartado anterior. De esta forma, se ha justificado el comportamiento de los ratios financieros, que miden unidireccionalmente el resultado de una actividad, y los precios de las acciones de ambas sociedades en el mercado de capitales chileno. El objeto que se persigue en el siguiente apartado es evaluar la gestión realizada en la región de Las Condes por el conjunto de empresas cotizadas y localizadas en la citada región, de acuerdo con las conclusiones alcanzadas en éste.

\section{ANALIISIS CUANTITATIVO DE LAS CLASES DE GESTION EN LA REGIÓN DE LAS CONDES}

Para realizar el análisis cuantitativo de las clases de gestión en la región chilena de Las Condes se ha procedido a obtener los valores medios de los indicadores de apalancamiento financiero (MRAF), suficiencia financiera (MSF) y de liquidez de transacción (MLT) para las distintas clases de gestión (CG) representadas en la Fig. 3 y recogidos en la Tabla 6 . Estos últimos indicadores miden el resultado de actividad en el área de tesorería (T), dado que esta área es la resultante financiera de la actividad realizada. De esta forma, el ratio MSFTCG-- representa la media de los ratios de suficiencia financiera en el área de tesorería para clases de gestión negativa y, así sucesivamente.

Tabla 6: Los indicadores de gestión de las clases de gestión en la región de Las Condes.

\begin{tabular}{|c|c|c|c|c|c|c|c|c|c|}
\hline Indicador $\left({ }^{*}\right)$ & 2014 & 2013 & 2012 & 2011 & 2010 & 2009 & 2008 & 2007 & 2006 \\
\hline M RAFCG-- & 0,7999 & 0,9544 & 0,6600 & 0,7426 & 0,7131 & 0,9391 & 1,0467 & $1,1284^{*}$ & $1,1997^{*}$ \\
\hline M RAFG-+- & 0,7432 & 0,7147 & 0,8081 & 0,6507 & 0,6671 & 0,6740 & 0,7821 & 1,0665 & 1,0812 \\
\hline M RAFCG ++ & $0,8442^{*}$ & $3,5514^{*}$ & 1,3990 * & $1,2505^{*}$ & $1,6528^{*}$ & $2,2256^{*}$ & $1,2014^{*}$ & 1,1018 & 1,1642 \\
\hline Año & 2014 & 2013 & 2012 & 2011 & 2010 & 2009 & 2008 & 2007 & 2006 \\
\hline M SFTCG-- & 0,8775 & 0,8270 & $0,9595^{*}$ & $1,0893^{*}$ & $0,9699^{*}$ & 0,7749 & $0,8564^{*}$ & $0,8826^{*}$ & $0,7798^{*}$ \\
\hline M SFTCG+- & 1,0585 & 0,8215 & 0,9576 & 1,0887 & 0,9695 & 0,7745 & 0,8561 & 0,8824 & 0,7796 \\
\hline M SFTCG++ & $1,1140^{*}$ & $0,8280^{*}$ & 0,8556 & 0,9190 & 0,7104 & $0,8859^{*}$ & 0,8460 & 0,8770 & 0,7768 \\
\hline Año & 2014 & 2013 & 2012 & 2011 & 2010 & 2009 & 2008 & 2007 & 2006 \\
\hline M LTTCG-- & 1,2283 & 1,3264 & 1,7795 & 2,6815 & 3,0824 & 3,6164 & 4,4311 & 4,7720 & 6,3868 \\
\hline M LTTCG+- & $0,6822^{*}$ & 0,7193 & 0,8542 & 1,2600 & 1,0491 & 0,8799 & 1,1034 & 1,4263 & 1,5101 \\
\hline M LTTCG ++ & 0,8820 & $0,5734^{*}$ & $0,6496 *$ & $0,6458^{*}$ & $0,7295^{*}$ & $0,6552^{*}$ & $0,5398^{*}$ & $0,6309^{*}$ & $0,6407^{*}$ \\
\hline
\end{tabular}

Los resultados de los valores medios de los indicadores de la Tabla 6 que tiene asignado un asterisco (*) son los que alcanzan mejor posición en ese ratio y para el año analizado. Los ratios de autonomía financiera (M RAF) son más elevados en las empresas que utilizan clases contables de gestión positivas y ofrecen mayor nivel de garantía al mercado que las otras dos clases de gestión. Este efecto, está asociado al nivel de liquidez de las transacciones. En las empresas de clase contables de gestión positiva el ratio (MLTTCG++), que mide el efecto interactivo entre las partidas de contables, ofrecen mejores resultados que en las otras dos clases de gestión, alcanzándose con mayor facilidad la condición de liquidez en las transacciones realizadas por las empresas. En la empresa PVR, para el periodo analizado, la relación entre los ratios de suficiencia financiera (MSF) y liquidez de transacción (MLT), estaba justificada y, en este 
apartado, vemos como la obtención de liquidez de transacción en las empresas con clase contable de gestión positiva, produce valores superiores a la unidad en el ratio de autonomía financiera, ofreciendo mayores garantías al mercado, relacionando el efecto financiero entre la gestión de clase positiva adoptada y la estructura financiera obtenida en la empresa PVR. Por otra parte, la evolución del ratio de suficiencia financiera del área de tesorería (MSFT) presenta mejor o peor resultado dependiendo de la clase de gestión y del año en que se produce las crisis financieras. En periodos de crisis financiera, años 2009 y 2013 , la mejor valoración se produce en las clases de gestión positivas y, en el resto de los años, la mejor valoración se produce en las empresas con gestión negativa. Además, las empresas que adoptan esta última clase de gestión obtienen una mejor evolución de su ratio de liquidez de transacción, indicando que debe haberse producido ajustes en la gestión para mejorar el efecto interactivo de las partidas contables que intervienen en la valoración de la liquidez de las transacciones.

Los resultados de la obtención de liquidez en el área de tesorería se presentan en la tabla 7. Las clases contables de gestión positiva presentan un mayor nivel en la condición de liquidez de transacción (CLTCG+) y el elevado volumen de sucesos o de clases contables de gestión que cumplen la condición de liquidez en el área de tesorería indica liquidez para la financiación externa obtenida y un mayor del ratio de autonomía financiera en la Tabla 6. Las empresas con clase contable de gestión negativa (CLTCG--) no cumplen la condición de liquidez y necesitan financiación ajena a la actividad. Sin embargo, en contraposición a lo indicado, las empresas que han adoptado clases contables de gestión negativas son las que han realizado mejor adaptación a las condiciones del entorno económico de la región de Las Condes, dado que presentan una minoración significativa del indicador MLTTCG-- que mide la liquidez de sus transacciones, en la tabla 6 , y se aproximan a cumplir la condición de liquidez de transacción. La minoración observada supone una corrección en los efectos interactivos de las partidas contables que intervienen en las transacciones contables. Las relaciones entre las clases contables de gestión y sus respectivas situaciones patrimoniales, se han analizado de forma individual para las empresas BSA y PVR en el apartado anterior, sobre las 9 clases contables de gestión desarrolladas anualmente por cada una de ellas, y en este apartado se han observado sobre las 156 clases de gestión obtenidas en la Tabla 5 para la región de Las Condes.

Tabla 7: Condiciones de liquidez del área de tesorería en las tres clases de gestión

\begin{tabular}{|l|c|c|c|c|c|c|c|c|c|}
\hline CONDICION LIQUIDEZ (tesorería) & 2014 & 2013 & 2012 & 2011 & 2010 & 2009 & 2008 & 2007 & 2006 \\
\hline CLTCG-- & 2 & 1 & 0 & 0 & 0 & 0 & 0 & 0 & 0 \\
\hline CLTCG+- & 3 & 2 & 2 & 2 & 0 & 2 & 1 & 1 & 1 \\
\hline CLTCG++ & 1 & 6 & 5 & 5 & 5 & 4 & 4 & 4 & 5 \\
\hline
\end{tabular}

\section{CONCLUSIONES}

En este artículo, continuación de otro anterior dedicado al análisis cualitativo de las empresas cotizadas chilenas, se ha realizado el análisis cuantitativo de las empresas cotizadas a través de la metodología contable de los gráficos radiales. El artículo contiene la aplicación material de la metodología contable de los gráficos radiales dirigida hacia un análisis financiero y las principales conclusiones se pueden resumir como siguen: a) efecto multidimensional de las decisiones de gestión adoptadas, b) las clases contables de gestión son un factor a considerar en la localización de las empresas, c) existe relación entre la clase contable de gestión adoptada, las fuentes de financiación de las empresas y la valoración de los mercados financieros y d) en periodos de crisis financiera las empresas con clase contable de gestión negativa han realizado mayor adaptación en su gestión que las empresas que han utilizado clases de gestión positiva y éstas últimas han ofrecido mayores garantías al mercado para el ejercicio continuado de su actividad. En consecuencia, es posible aseverar que las clases contables de gestión existentes un entorno económico son un factor a considerar en el estudio de localización de las empresas y que las decisiones adoptadas en un área de gestión tienen repercusión en el conjunto de la actividad empresarial, constituyendo ambos aspectos son base para la elaboración de futuras líneas de investigación.

\section{REFERENCIAS}

Ascani, A., R. Crescenzi y S. lammarino, New Economic Geography and Economic Integration: A Review, WP1/02 Search Working Paper. Universidad de Barcelona. (En la web: http://www.ub.edu/searchproject/, acceso: 8 de abril de 2016). January (2012)

Bolsa de Santiago, (CTodos los derechos reservados. https://goo.gl/zuVbv4. Acceso: 16 de mayo (2016)

Buchman, T.A., P. Harris y M. Liu, GAAP VS. IFRS treatment of leases and the impact on financial ratios, Review of Business and Finance Studies, ISSN: 2156-8081, 7(1), 93-104 (2016) 
Cárdenas, U. y J. Ojeda, La nueva economía institucional y la teoría de la implementación, Revista de Economía Institucional, ISSN: 0124-5996, 4(6), 153-169, http://www.economiainstitucional.com (2008)

Dimitrios, B., E. Nikolaos, P. Konstantinos, y V. Dimitrios, The impact of IFRS on ratios of listed and new listed companies of Athens Exchange, Inter. Journal of Business and Social Research, 3(5), 149-157 (2013)

García Garnica, A., y E.L. Taboada Ibarra, Teoría de la empresa: las propuestas de Coase, Alchian y Demsetz, Williamson, Penrose y Nooteboom, Economía: teoría y práctica, 36, 9-42 (2012)

Geyskens, I., J.B.E. Steenkamp y N. Kumar, Make, buy, or ally: A transaction cost theory meta-analysis. Academy of management journal (en línea), 49(3), 519-543, https://goo.gl/GiAUjY (2006)

Ibiamke, N.A., y P.B. Ateboh-Briggs, Financial ratios effect of international financial reporting standards (IFRS) adoption in Nigeria, Journal of Business and Management Invention, 3(3), 50-59 (2014)

Lantto, A.M., y P. Sahlström, Impact of International Financial Reporting Standard adoption on key financial ratios. Accounting and Finance, 49(2), 341-361 (2009)

Martínez-Ochoa, L. y M.A. Pérez-Benedito, Análisis de Estrategias de Gestión: Aplicaciones Polivalentes Del Gráfico Radial, Revista Iberoamericana de Contabilidad de Gestión, 9(17), Enero-Junio (2011)

Pérez-Benedito, M.A., Comparative analysis of enterprises of covering floors and walls by applying the radar charts methodology, doi: 10.11648/j.sjbm.20130104.14, Science Journal of Business and Management, 1(4), 81-87 (2013)

Pérez-Benedito, M.A., L. Porcuna-Enguix y R. Porcuna-Eguix, Los Mapas Contables de Gestión de las Empresas Cotizadas Chilenas: Análisis Cualitativo, Información Tecnológica, ISSN 0718-0764, 28(1), 161$170(2017)$

Pérez -Benedito, M.A., The Analysis of Behavior of Switzerland Company by Methodology of Radar Chart, European Journal of Business and Social Sciences, ISSN: 2235-767X, 3(6), 136-155, September (2014)

Pérez -Benedito, M.A., The Application of Accounting Methodology of Radar Charts to Analyze the Sector of Sawmilling and Planning of Wood of Austria, doi: 10.14738/abr.34.1254, Archives of Business Research, 3(4), 1-14 (2015)

Salgado, E., Teoría de costos de transacción; una breve reseña. Cuadernos de Administración, (en línea), 16(26), 61-78, https://goo.gl/hmwntk, acceso 8 de abril (2003)

Sobrino, J., Localización industrial y concentración geográfica en México, Estudios Demográficos y Urbanos de El Colegio de México, ISSN 2448-6515, 31(1), 9-56 (2016)

Terzi, S., R. Oktem, y I.K. Sen, Impact of adopting international financial reporting standards: empirical evidence from Turkey, International Business Research, 6(4), 55-66 (2013)

Urbano, D., J.C. Díaz y R. Hernández, Evolución y principios de la Teoría Económica Institucional. Una propuesta de aplicación para el análisis de los factores condicionantes de la creación de empresas. Investigaciones Europeas de Dirección y Economía de la Empresa, ISSN: 1135-2533, 13(3), 183-198 (2007) 